\title{
Uso de redes sociales en estudiantes de Promoción de la Salud en la era de la COVID-19
}

\author{
Use of Social Networks in Health Promotion Students in the era of COVID-19
}

\author{
Elizabeth Espinoza-Portilla « 1,a, Patricia Pimentel-Álvarez 1,b, Américo Peña-Oscuvilca 1,c, Félix Llanos-Tejada 1,d
}

Filiación y grado académico

Universidad Continental, Lima, Perú.

Doctora en Gobierno y Políticas Públicas.

Maestra en Salud Pública.

Médico Cirujano especialista en Cirugía de Tórax y Cardiovascular.

Médico Cirujano especialista en Neumología.

19. ORCID iD de Elizabeth Espinoza-Portilla https://orcid.org/0000-0002-5068-5357

16) ORCID iD de Patricia Pimentel-Álvarez https://orcid.org/0000-0001-7299-1227

10 ORCID iD de Américo Peña-Oscuvilca https://orcid.org/0000-0003-4026-9832

10 ORCID iD de Félix Llanos-Tejada

https://orcid.org/0000-0003-1834-1287

Contribución de los autores

EEP: concepción y diseño de la investigación, re colección de datos, análisis de datos, redacción de manuscrito, revisión crítica del manuscrito aprobación de la versión final.

PPA: revisión crítica y redacción del artículo, aprobación de la versión final.

APO: revisión crítica y redacción del artículo, aprobación de la versión final.

FLLT: revisión crítica y redacción del artículo aprobación de la versión final.

Fuentes de financiamiento

La investigación fue realizada con recursos propios.

Conflictos de interés

Los autores declaran no tener conflicto de interés.

Recibido: 08-06-2021

Arbitrado por pares

Aceptado: 01-10-2021

Citar como

Espinoza-Portilla E, Pimentel-Álvarez P, Peña-Oscuvilca A, Llanos-Tejada F. Uso de redes sociales en estudiantes de Promoción de la Salud en la era de la COVID-19. Rev Perú Cienc Salud. 2021; 3(4): 24855. doi: https://doi.org/10.37711/rpcs.2021.3.4.357

Correspondencia

Elizabeth Espinoza-Portilla

Dirección: Calle Junín 355, Miraflores 15046,

Lima, Perú.

Correo: espinoza.portilla@gmail.com

\section{RESUMEN}

Objetivo. Describir el uso de las redes sociales (TikTok y Twitter) en estudiantes de medicina de una universidad privada peruana como parte de la actividad grupal de la primera evaluación de la asignatura de Promoción de Salud-Determinantes. Métodos. Estudio descriptivo y transversal, con enfoques cuantitativo y cualitativo. Se incluyeron a todos los estudiantes (60) registrados en la asignatura de Promoción de Salud-Determinantes. Como parte de la actividad grupal de la primera evaluación de la asignatura se les asignó a los estudiantes grabar un video corto en grupos, el cual luego debía ser compartido en las plataformas TikTok y Twitter. Resultados. Los estudiantes realizaron y compartieron satisfactoriamente en redes sociales 10 videos sobre los siguientes tópicos: promoción de la vacunación COVID-19, promoción sobre el uso del preservativo para prevenir infecciones de transmisión sexual, prevención del embarazo adolescente, promoción del uso de mascarillas y promoción de la vasectomía. Asimismo, los estudiantes brindaron comentarios positivos sobre la experiencia y se discutieron las principales características de la información en salud o las estrategias de comunicación en salud utilizadas en base a los tópicos asignados. Conclusiones. Los estudiantes lograron satisfactoriamente el resultado de aprendizaje, ya que fueron capaces de explicar los objetivos, principios y elementos fundamentales de la información en salud y estrategia de comunicación en salud, a través del tópico asignado.

Palabras clave: promoción de la salud; educación médica; red social; Twitter TikTok; Perú (fuente: DeCS BIREME).

\section{ABSTRACT}

Objective. The objective of this article is to describe the use of the social networks (TikTok and Twitter) in medical students at a private Peruvian university, as part of the group activity for the first evaluation of the course: Health Promotion - Determinants. Methods Descriptive and cross-sectional study, with a quantitative and qualitative focus. All students (60) registered in the course: Health Promotion - Determinants were included. As part of the group activity for the first evaluation, the students were assigned to record a short video which then had to be shared on the TikTok and Twitter platforms. Results. The students made and shared on social networks (TikTok and Twitter) 10 videos on the following topics: promotion of COVID-19 vaccination, promotion of the use of condoms to prevent sexually transmitted infections, prevention of adolescent pregnancy, promotion of use mask and vasectomy promotion. Conclusions. We consider that the promotion of a variety of social networks and digital resources can help the teaching-learning process and the understanding of an increasingly digitized health promotion.

Keywords: health promotion; education, medical; social networking; Twitter; TikTok; Peru (source: MeSH NLM). 


\section{INTRODUCCIÓN}

La complejidad del cuidado de la salud en el siglo XXI, aún más con desafíos como la pandemia por la COVID-19, requiere repensar los paradigmas educativos médicos actuales ${ }^{(1)}$, ya que los estudiantes de Medicina de pregrado provienen de varias generaciones: los tradicionalistas, los baby boomers, la generación $\mathrm{X}$, la generación $\mathrm{Y}$ o millenials y la generación $Z$ o centenials ${ }^{(2)}$. Los estudiantes de Medicina están cada vez más familiarizados y son capaces de integrar tecnologías de rápida evolución en todos los aspectos de sus vidas ${ }^{(3)}$.

Por ello, es necesario diseñar nuevas estrategias pedagógicas, o mejorar las existentes, de modo que puedan ser más atractivas a los estudiantes $y$, al mismo tiempo, abordar las demandas de los paradigmas de educación médica del siglo XXI ${ }^{(3)}$. En otras palabras, las estrategias pedagógicas, como las técnicas didácticas de enseñanza, deben complementarse con estrategias pedagógicas particularmente centradas en las nuevas generaciones de los estudiantes de Medicina ${ }^{(4)}$.

Por otro lado, las redes sociales hacen referencia a sitios web y aplicaciones que permiten a los usuarios crear y compartir contenido, interactuar con otros usuarios o encontrar personas con intereses similares ${ }^{(3)}$. El término cubre múltiples plataformas que abarcan blogs y microblogs como Twitter, Wikis, YouTube y sitios de redes sociales como Facebook ${ }^{(5)}$. La integración de las aplicaciones de redes sociales en las estrategias pedagógicas para los estudiantes de Medicina de las nuevas generaciones no solo abordará sus necesidades de aprendizaje, sino que también atraerá el estilo o la estrategia de aprendizaje preferidos por los estudiantes de Medicina (3). El fundamento de lo anterior se encuentra respaldado por la teoría del aprendizaje del conectivismo ${ }^{(3)}$.

El conectivismo considera el aprendizaje como un proceso multifacético catalizado por la tecnología y la socialización ${ }^{(6)}$. Los cimientos del conectivismo están alimentados, entre otros, por la conectividad, la complejidad y la autoorganización. Los principios del conectivismo ${ }^{(6,7)}$ a destacar son los siguientes:

- El aprendizaje y el conocimiento descansan en la diversidad de perspectivas.

- El aprendizaje es un proceso de conectar nodos especializados o fuentes de información.

- Es necesario fomentar y mantener las conexiones para facilitar el aprendizaje continuo.

- La capacidad de ver conexiones entre campos, ideas y conceptos es una habilidad fundamental.
- La toma de decisiones es en sí misma un proceso de aprendizaje. La elección de qué aprender y el significado de la información entrante se ve a través de la lente de una realidad cambiante.

Por lo tanto, los docentes o instructores en un entorno de aprendizaje conectivista guían a los estudiantes hacia la información (a la que preferiblemente se puede acceder con facilidad) y responden las consultas según sea necesario, para alentar el aprendizaje y el intercambio de los estudiantes por su cuenta a través de la creación de una comunidad de aprendizaje ${ }^{(3)}$. También se anima a los estudiantes a buscar información en la web, a criticar la información y compartir sus hallazgos y opiniones dentro de la comunidad de aprendizaje que han creado $^{(3)}$.

Existen reportes de uso de varias plataformas de redes sociales, así como de numerosos y variados instrumentos digitales dentro de la educación médica de pregrado, dentro de los planes de estudio de educación médica de pregrado ${ }^{(8)}$, relacionados al profesionalismo en línea ${ }^{(9)}$, el aprendizaje blended ${ }^{(10)}$ (que combina horas presenciales y virtuales en una misma asignatura), el bienestar de los estudiantes ${ }^{(11)}$ y la mentoría ${ }^{(12)}$ que representan una fracción de todas las aplicaciones de las redes sociales en el pregrado de Medicina.

El argumento para la integración de las redes sociales en la práctica educativa está respaldado por múltiples puntos de vista teóricos ${ }^{(8)}$. Como educadores, ya no se trata de saber si las redes sociales tienen aplicaciones educativas o si las poblaciones de estudiantes universitarios están utilizando activamente estas plataformas para su aprendizaje. Al contrario, deberíamos preguntarnos cuál es la mejor manera de utilizar las redes sociales y si dichas plataformas pueden facilitar resultados específicos $y$, por ende, ayudarnos a cumplir los resultados de aprendizaje planteados ${ }^{(8)}$. En ese sentido, existen revisiones sistemáticas sobre el uso e impacto de las redes sociales en la educación médica que han investigado diversos desenlaces y su eficacia ${ }^{(8,13-15)}$.

Con el advenimiento de la pandemia por la COVID-19, la comunidad de educación médica se ha transformado a nivel global ${ }^{(16)}$. Goh y Sandars hacen un llamado a un nuevo paradigma de educación médica a través del aprendizaje mejorado por la tecnología ${ }^{(17)}$, considerando a las tecnologías emergentes como la inteligencia artificial y las analíticas de aprendizaje. Asimismo, se necesitan nuevos enfoques para mejorar la educación de los profesionales de la salud ${ }^{(18)}$. Por ello, las redes sociales y las aplicaciones tecnológicas pueden ser una herramienta útil para facilitar el contacto entre los estudiantes y apoyar el aprendizaje activo ${ }^{(18)}$. 
Por consiguiente, el objetivo de la investigación es describir el uso de las redes sociales (TikTok y Twitter) en estudiantes de Medicina de una universidad privada peruana como parte de la actividad grupal de la primera evaluación de la asignatura de Promoción de SaludDeterminantes.

\section{MÉTODOS}

\section{Tipo de estudio}

Se realizó un estudio descriptivo y transversal. El estudio aborda un componente cuantitativo y se complementa con comentarios cualitativos relacionados a las percepciones de los estudiantes sobre la actividad grupal como parte de la asignatura.

\section{Población y muestra}

Se incluyeron a todos los estudiantes (60) registrados en la asignatura de Promoción de Salud-Determinantes de la E.A.P. de Medicina filial Lima (EAPML) de la Universidad Continental, licenciada por la Superintendencia Nacional de Educación Superior Universitaria el año 2018.

\section{Descripción de la asignatura}

La asignatura de Promoción de Salud-Determinantes es de tipo obligatoria y de carácter teórico-práctico, encontrándose en el currículo del octavo periodo. La relevancia de la asignatura reside en: "analizar y aplicar el modelo de abordaje de promoción de la salud para los determinantes sociales de la salud" (19). Los contenidos que desarrolla la asignatura son:"promoción de la salud (enfoques, estrategias, etapas de vida, escenarios y ejes temáticos) y determinantes sociales de la salud (globalización; contexto sociopolítico y económico; sistema de salud, determinantes estructurales de la salud, determinantes intermedios de la salud, impacto en la salud y propuestas de implicancia política)"(19).

\section{Instrumentos de recolección de datos}

Se registró el número de reproducciones de cada video compartido por los estudiantes a través de las cuentas de redes sociales (TikTok y Twitter) utilizando una hoja de cálculo.

Asimismo, se registraron comentarios selectos que se realizaron durante la presentación de los videos en la sesión correspondiente (semana 5) de la asignatura.

\section{Procedimientos de la recolección de datos}

La asignatura comenzó a impartirse a partir del segundo semestre del 2021 y, debido a la pandemia por la COVID-19, se tuvo que adaptar a la modalidad virtual. En la asignatura de la EAPML se registraron 60 estudiantes, los cuales se dividieron en dos secciones de 30 estudiantes cada uno para el componente teórico; además, se dividieron en seis secciones de 10 estudiantes cada uno para el componente práctico. La asignatura se encuentra organizada con las siguientes unidades:

- Unidad 1: marco conceptual, metodología, enfoques y estrategias de la educación para la salud.

- Unidad 2: ejes temáticos y escenarios de la educación para la salud.

- Unidad 3: determinantes sociales de la salud, componente sociopolítico y estructural.

- Unidad 4: determinantes sociales de la salud, impacto de la salud y propuestas de implicancia política.

Como parte de los contenidos y actividades propuestas se utilizaron las "metodologías activas y el trabajo colaborativo, promoviendo el debate con discusiones y diálogos simultáneos, métodos de casos, panel, trabajo en equipo, además del chat y el foro a través del aula virtual". Los estudiantes realizaron trabajos en equipo, fomentando la investigación con el uso de herramientas digitales disponibles en Internet y se complementaron con audios, videos y recursos multimedia, propiciando el "desarrollo de comunidades de interaprendizajes" mediados por la tecnología ${ }^{(19)}$. Todos los estudiantes interactúan y hacen uso del aula virtual provista por la Universidad Continental. Asimismo, se han utilizado diversas plataformas y aplicaciones tecnológicas que serán detalladas en una próxima comunicación y que han logrado que, junto con las redes sociales, los docentes se adapten al mundo digital de los estudiantes.

\section{Actividad grupal de la primera evaluación de la asignatura}

Como parte de la actividad grupal de la primera evaluación de la asignatura (consolidado 1), se les asignó a los estudiantes grabar un video corto en grupos de seis personas el cual luego debería ser compartido en las plataformas TikTok (https://www.tiktok.com/) y Twitter (https://www.twitter.com/).

Uno de los resultados de aprendizaje esperados fue que, al culminar la actividad grupal y en base a la información brindada durante las sesiones teóricas y prácticas, los estudiantes sean capaces de explicar los objetivos, principios y elementos fundamentales de la información en salud y estrategia de comunicación en salud, a través del tópico asignado.

Previamente, a los estudiantes se les brindó pautas y recomendaciones para dicho proceso. Resulta fundamental 
que los estudiantes cumplan con los estándares éticos y legales básicos acerca del uso de redes sociales en salud, incluyendo la importancia de resguardar la confidencialidad de las personas, el consentimiento informado, etc. La importancia de estas recomendaciones para compartir información de salud en las redes sociales se ha vuelto particularmente evidente durante la pandemia de la COVID-19 porque algunas plataformas de redes sociales detectan publicaciones sobre la COVID-19 y recomiendan que los usuarios que ven estas publicaciones visiten los sitios web de las autoridades de atención médica para obtener información precisa.

Los tópicos de promoción de la salud seleccionados fueron:

\section{- Promoción de la vacunación COVID-19.}

- Promoción sobre el uso del preservativo para prevenir infecciones de transmisión sexual.

- Prevención del embarazo adolescente.

- Promoción del uso de mascarillas.

- Promoción de la vasectomía.

Se recopilaron los enlaces web de los videos compartidos a través de las cuentas de redes sociales en una ficha, los que fueron registrados por los estudiantes en el aula virtual de la asignatura, y se consignó el número de reproducciones de cada video.

Asimismo, se registraron en una ficha de observación los comentarios realizados por los estudiantes, de manera voluntaria, en relación a su experiencia y percepción durante el proceso de realización de video e interacción con las redes sociales, se recolectaron dichos comentarios durante la sesión virtual de la semana 5 , y se codificaron protegiendo la identidad de los estudiantes.

\section{Análisis de datos}

Para el análisis de los datos cuantitativos se utilizaron métodos descriptivos numéricos y porcentajes. La docente coordinadora del curso analizó el contenido de cada video (disponible en el aula virtual de la asignatura) utilizando una rúbrica previamente establecida en la guía de aprendizaje de la asignatura. Los datos cualitativos, recogidos a través de los comentarios que expresaron los estudiantes durante las presentaciones de los videos, se analizaron mediante la técnica de análisis de contenidos de las narrativas de los estudiantes, de acuerdo con el modelo de la teoría fundada, y se seleccionaron frases destacadas, los cuales se presentan en la sección resultados.

\section{Aspectos éticos}

Para el estudio se consideraron principios bioéticos aplicados en la investigación incluidos en la Declaración de Helsinki de la Asociación Médica Mundial ${ }^{(20)}$. Asimismo, si bien para la producción y preparación del video era necesario la participación de todos los miembros del grupo, la participación y aparición de los estudiantes en los videos fue voluntaria.

\section{RESULTADOS}

En cuanto a las características de los estudiantes registrados en la asignatura, aproximadamente un tercio de estos eran hombres y uno de cada cinco estudiantes ya contaba con una primera carrera profesional vinculada al campo de la salud (ver Tabla 1). Es importante precisar que cada sección de estudiantes (sección 1 y 2) realizó y compartió cinco videos cada una.

Así, se registraron 10 videos durante la primera evaluación de la asignatura, considerando dos videos por cada tópico seleccionado. En la tabla 2 se muestran los enlaces web a los videos, y se incluye el número de reproducciones de cada uno.

\section{Comentarios de los estudiantes}

A continuación, se destacan algunos comentarios de los estudiantes sobre la experiencia de elaborar y compartir los videos sobre promoción de la salud realizados durante la asignatura. En general, percibieron la experiencia como positiva y destacaron como características más saltantes: mayor conocimiento del tópico de promoción de la salud asignado, mayor información sobre las características de mensajes efectivos en comunicación en salud (como dibujos animados y elementos musicales) y mayor conocimiento sobre el estilo documental de los videos elaborados y compartidos en las redes sociales.

Tabla 1. Características de los estudiantes de Promoción de SaludDeterminantes

\begin{tabular}{|c|c|c|c|c|}
\hline \multirow{3}{*}{ Características } & \multicolumn{4}{|c|}{$n=60$} \\
\hline & \multicolumn{2}{|c|}{ Sección 1} & \multicolumn{2}{|c|}{ Sección 2} \\
\hline & fi & $\%$ & fi & $\%$ \\
\hline \multicolumn{5}{|l|}{ Sexo } \\
\hline Mujer & 20 & 33,3 & 20 & 33,3 \\
\hline Hombre & 10 & 66,7 & 10 & 66,7 \\
\hline \multicolumn{5}{|c|}{ Cuenta con primera carrera profesional } \\
\hline Sí & 7 & 23,3 & 5 & 16,7 \\
\hline Total & 30 & 100,0 & 30 & 100,0 \\
\hline
\end{tabular}


Tabla 2. Enlaces web y número de reproducciones de los videos producidos y realizados por los estudiantes y compartidos vía TikTok y Twitter

\begin{tabular}{|c|c|c|}
\hline Tópicos asignados & $\begin{array}{l}\text { Enlace del video-Sección } 1 \\
\text { (número de reproducciones) }\end{array}$ & $\begin{array}{l}\text { Enlace del video-Sección } 2 \\
\text { (número de reproducciones) }\end{array}$ \\
\hline Grupo 1: promoción de la vacunación COVID-19 & $\begin{array}{l}\text { https://bit.ly/3Atjm6Q } \\
\text { ( } 2700 \text { reproducciones) }\end{array}$ & $\begin{array}{l}\text { https://bit.ly/3hT52gE } \\
\text { (289 reproducciones) }\end{array}$ \\
\hline $\begin{array}{l}\text { Grupo 2: promoción sobre el uso del preservativo } \\
\text { para prevenir infecciones de transmisión sexual }\end{array}$ & $\begin{array}{l}\text { https://bit.ly/3nQCMyY } \\
\text { (46 reproducciones) }\end{array}$ & $\begin{array}{l}\text { https://bit.ly/39nLUmb } \\
\text { (357 reproducciones) }\end{array}$ \\
\hline Grupo 3: prevención del embarazo adolescente & $\begin{array}{l}\text { https://bit.ly/3nUwb6s } \\
\text { (389 reproducciones) }\end{array}$ & $\begin{array}{l}\text { https://bit.ly/2XCguWp } \\
\text { (228 reproducciones) }\end{array}$ \\
\hline Grupo 4: promoción del uso de mascarillas & $\begin{array}{l}\text { https://bit.ly/2XAzWCY } \\
\text { (77 reproducciones) }\end{array}$ & $\begin{array}{l}\text { https://bit.ly/3EBOTFX } \\
\text { (205 reproducciones) }\end{array}$ \\
\hline Grupo 5: promoción de la vasectomía & $\begin{array}{l}\text { https://bit.ly/3lOcFpC } \\
\text { (76 reproducciones) }\end{array}$ & $\begin{array}{l}\text { https://bit.ly/2Xz4wgz } \\
\text { (560 reproducciones) }\end{array}$ \\
\hline
\end{tabular}

Algunos comentarios positivos fueron: "Ha sido muy divertido grabar el video en TikTok. Nos tratamos de enfocar no sólo en la información sino le dimos un tono un tanto gracioso para que la gente se quede un poco entretenida, pero a la vez se lleve el mensaje de que debe vacunarse. Al final de todo, nos gustó poder concientizar, pero de manera divertida"; "la experiencia fue graciosa y académica, ya que nos hemos informado más sobre el tópico asignado"; "la experiencia ha sido bonita. Colaboramos entre todos para armar el video y subirlo. Hemos compartido y aprendido mucho"; "hemos tratado de hacer (el video) lo más didáctico posible, dinámico y divertido a la vez para causar un mayor impacto".

\section{DISCUSIÓN}

Si bien existe evidencia que resalta la importancia del uso de redes sociales en medicina, incluyendo Twitter para promover la educación continua y la investigación en salud en el Perú ${ }^{21,22)}$, no encontramos literatura sobre su uso como parte del currículo en medicina a nivel de pregrado. Por ello, hasta nuestro conocimiento, reportamos la primera experiencia del uso de las herramientas TikTok y Twitter para actividades académicas en el contexto de una asignatura universitaria de Promoción de la Salud en el Perú.

Los estudiantes, previamente a la realización de los videos, ya contaban con una cuenta en Twitter y estaban familiarizados con el uso de TikTok. Sin embargo, la mayoría no había creado ni generado contenidos sobre salud. Así, como parte de las sesiones teóricas de la asignatura, se mostraron ejemplos de cómo las redes sociales, en específico TikTok, se utilizan como herramientas para la promoción de salud ${ }^{(23)}$. Consideramos que, al finalizar la actividad grupal descrita en este artículo, los estudiantes lograron satisfactoriamente el resultado de aprendizaje, ya que fueron capaces de explicar los objetivos, principios y elementos fundamentales de la información en salud y la estrategia de comunicación en salud a través del tópico asignado.

Existen algunas características por las que, a diferencia de otras plataformas de redes sociales, TikTok destaca como la posibilidad de crear micro-videos cortos con funciones de edición e inclusión de música fáciles de usar $^{(24,25)}$. La aplicación de TikTok proporciona una interfaz de usuario no complicada para crear videos en donde los usuarios pueden incrustar fácilmente sus opciones de música preferidas y efectos especiales y donde los videos se almacenan ${ }^{(25)}$. Zhu et al. ${ }^{(23)}$ sugieren que TikTok debe verse como un medio oficial para comunicar información en salud a los ciudadanos y no solamente como un canal de entretenimiento; así, debe convertirse en una parte integral de los ecosistemas de redes sociales de las instituciones de salud, permitiendo que las instituciones interactúen con los ciudadanos a un nivel más personal, extendiendo la comunicación más allá de la forma meramente escrita.

En el sector de la salud, la adopción de las redes sociales no es nueva ${ }^{(26-28)}$. Los medios sociales han sido ampliamente adoptados por pacientes, familiares o cuidadores y profesionales sanitarios, y numerosos estudios reportan su utilidad para el empoderamiento del paciente, la promoción de la salud, la vigilancia de la salud pública y la mejora de la calidad en salud ${ }^{(29-32)}$. Sin embargo, otros estudios se han centrado en revelar el lado oscuro de las redes sociales en la salud. Por ejemplo, al examinar cómo el contenido no verificado conduce al intercambio de información engañosa y puede promover la desinformación en salud ${ }^{(3) \text {; }}$; por otro lado, los pacientes se pueden volver demasiado confiados en su propia toma de decisiones médicas ${ }^{(34-35)}$ y existen desafíos en relación con los temas de privacidad, confidencialidad y seguridad de la información ${ }^{(36-37)}$.

La comunicación eficaz de información sanitaria a través de las redes sociales requiere, eso sí, de un número 
considerable de seguidores (followers). Nuestro estudio encontró que el video que más reproducciones tuvo fue compartido por un estudiante de Medicina de la asignatura que tenía un gran número de seguidores en la red social Twitter (más de 41,300 seguidores). Esto sugiere que el impacto de los mensajes puede ser masificado y potenciado contando como aliados a personas con muchos seguidores y de los denominados influencers. Asimismo, los estudiantes de la asignatura utilizaron elementos dentro de sus videos como incorporar canciones muy populares para potenciar el impacto de sus videos. Este elemento de incorporar música popular se ha identificado como un elemento que aporta valor a los mensajes por autores como Zhu et al. ${ }^{(23)}$.

Con respecto al tipo de contenido, se observó que algunos videos contenían disfraces realizados con materiales fáciles de conseguir y otros videos se presentaban a manera de documental mostrando contenidos relacionados con determinado tópico de salud pública. Esto concuerda con la noción de que el enfoque narrativo de contar historias se ha adoptado cada vez más para difundir contenido relacionado con la salud en la comunicación sanitaria ${ }^{(38)}$.

Consideramos que TikTok es una valiosa comunidad de cocreación donde las instituciones de salud pueden promover estilos de vida saludables y brindar información relacionada con la salud, mientras que los usuarios también pueden generar información relacionada con la salud para impactar a otras personas, incluyendo autoridades y políticos. Y sobre todo llega a una población que no necesariamente puede estar interesada en acceder a los noticieros, periódicos o blogs de noticias, lo cual lo hace mucho más interesante, porque se está formando una generación no sólo creativa sino preocupada y activa por la salud pública, y no egoísta como muchos medios nos podrían hacer creer, así se podría enfocar como la reivindicación de esta generación.

Sin embargo, es muy importante reforzar que el comportamiento en redes sociales de los estudiantes de Medicina (y de los profesionales de salud en general) debe ser siempre profesional ${ }^{(36)}$. Así, es importante respetar la privacidad y el buen nombre, además del comportamiento deontológicamente correcto, los cuales son obligaciones constantes en las redes sociales ${ }^{(36)}$. Los estudiantes de Medicina deben considerar cuidadosamente qué información compartir en las redes sociales y cómo compartirla. Además, los estudiantes deben asumir la responsabilidad del contenido proporcionado como lo haría cualquier profesional de la salud.
Uno de los muchos beneficios de las redes sociales es la capacidad de compartir información sobre una enfermedad o afección con la población en general de manera accesible. Las redes sociales se pueden utilizar para crear conciencia, combatir la información errónea, brindar apoyo al paciente, responder preguntas comunes, interactuar con los pacientes y más. Resulta esencial que los estudiantes de Medicina proporcionen fuentes confiables de información para referencia pública. Por ejemplo, en la asignatura presentada en este estudio se les proporcionó a los estudiantes fuentes confiables de Internet, como MedlinePlus ${ }^{\circledR}$ de la Biblioteca Nacional de Medicina de los EE.UU. ${ }^{(39)}$.

Este estudio tiene, sin embargo, ciertas limitaciones. En primer lugar, se trató de un estudio descriptivo que exploraba el uso de la plataforma TikTok por un grupo de estudiantes de Medicina, en el contexto evaluativo de una asignatura de medicina. Para este estudio no consideramos el número de mensajes compartidos y tampoco consideramos los comentarios a los videos. También, se requiere una mayor duración del periodo de evaluación para ver más dinámicas de estas plataformas de redes sociales. Las investigaciones futuras pueden ampliar la muestra del estudio utilizando técnicas automatizadas, por ejemplo, el análisis de sentimientos o análisis narrativo para revelar una imagen más completa de las cuentas de TikTok analizadas.

En segundo lugar, nuestro estudio no examinó los cambios de comportamiento relacionados con la salud que resultaron de visualizar los videos en TikTok, por lo que una comparación adicional del efecto en la salud a través de diferentes canales de redes sociales podría ser de gran valor para comprender el impacto de las redes sociales en salud.

Además, es necesario una mayor exploración de los estudiantes que elaboraron los videos de salud a través de TikTok lo que merece mayor atención. Por ejemplo, realizar estudios cualitativos que analicen sus percepciones y actitudes de interactuar con TikTok y sus expectativas sobre el contenido. En ese sentido, es posible que los estudiantes no necesariamente hayan adquirido todas las habilidades y estrategias para la comunicación de la salud a través de estos canales emergentes de las redes sociales.

Es importante destacar la experiencia previa adquirida por los docentes participantes de esta asignatura en el uso de redes sociales y plataformas virtuales, que fue fortalecida con sesiones y cursos de inducción que recibieron por el área académica de la universidad, previo 
al inicio de la asignatura. Así, en cada una de las sesiones de esta asignatura se promovieron y utilizaron los hashtags en Twitter: \#PromocionDeLaSalud y \#SaludParaTodos para compartir mensajes relevantes sobre promoción de la salud en esta red social.

Adicionalmente, los docentes de esta asignatura (EEP, PPA, APO, y FLLT) utilizaron las siguientes herramientas digitales durante las sesiones: Jamboard, Kahoot!, Slido, la funcionalidad de encuestas incorporada en Google Meet, y Whatsapp, para coordinaciones entre los docentes y los estudiantes coordinadores de la asignatura, en adición a la herramienta de Google Meet. Como anotan Giraldo Ospina et al. las condiciones de "agilidad, inmediatez y flexibilidad que ofrecen las tecnologías digitales", especialmente las redes sociales, favorecen y permiten dar continuidad a las actividades académicas y administrativas en tiempos de la COVID-19 ${ }^{(40)}$.

Existen muchos retos por superar para lograr una promoción de salud digitalizada y una atención primaria en salud digitalizada en el Perú, las cuales se han descrito previamente en la literatura nacional e internacional ${ }^{(41-42)}$.

En conclusión, en este artículo se ha descrito el uso de las redes sociales (TikTok y Twitter) en estudiantes de Medicina de una universidad privada peruana como parte de la actividad grupal de la primera evaluación de la asignatura de Promoción de Salud-Determinantes. Consideramos que, al finalizar la actividad grupal descrita en este artículo, los estudiantes lograron satisfactoriamente el resultado de aprendizaje, ya que fueron capaces de explicar los objetivos, principios y elementos fundamentales de la información en salud y estrategia de comunicación en salud, a través del tópico asignado. Además, la inclusión y promoción del uso de una variedad de redes sociales y recursos digitales puede ayudar al proceso de enseñanza-aprendizaje y a la comprensión de una promoción de la salud cada vez más digitalizada. Por último, estos resultados pueden servir como referencia para otras asignaturas relacionadas a salud pública a nivel universitario, tanto nacionales como internacionales.

Agradecimientos. Los autores expresan su agradecimiento a los estudiantes de la asignatura, por su creatividad, activa participación y la capacidad de trabajo en equipo demostrada durante la actividad de grabación, edición y difusión de los videos.

\section{REFERENCIAS}

1. Rizk AA, Acharya N, Elzawy M, Hana K, Elzawy G. An analysis of medical education suggestions and inter- ventions during the COVID-19 pandemic: A literature review. University of Toronto Medical Journal 2021; 98(3): 78-87.

2. Ortega-Miranda EG. Mentoría entre pares en la educación médica de pregrado como herramienta para mejorar el aprendizaje y responder a las demandas de las nuevas generaciones. Acta Méd Peru, 2019; 36(1): 57-61.

3. Banerjee Y, Tambi R, Gholami M, Alsheikh-Ali A, Bayoumi R, Lansberg P. Augmenting Flexnerism Via Twitterism: Need for Integrating Social Media Application in Blueprinting Pedagogical Strategies for Undergraduate Medical Education. JMIR Med Educ. 2019; 5(1): e12403.

4. Tanaka M, Mizuno K, Fukuda S, Tajima S, Watanabe Y. Personality traits associated with intrinsic academic motivation in medical students. Med Educ. 2009; 43(4): 384-387.

5. Katz M, Nandi N. Social Media and Medical Education in the Context of the COVID-19 Pandemic: Scoping Review. JMIR Med Educ. 2021; 7(2): e25892. https://doi. org/10.2196/25892

6. Goldie JG. Connectivism: a knowledge learning theory for the digital age? Med Teach. 2016; 38(10): 1064-1069.

7. Downes S. New technology supporting informal learning. J Emerge Tech Web Intell. 2010; 2(1): 27-33.

8. Guckian J, Utukuri M, Asif A, Burton O, Adeyoju J, Oumeziane $A$, Chu T, Rees EL. Social media in undergraduate medical education: A systematic review. Med Educ. 2021. https://doi.org/10.1111/medu.14567

9. Kaczmarczyk JM, Chuang A, Dugoff L, Abbott JF, Cullimore AJ, Dalrymple J, et al. e-Professionalism: a new frontier in medical education. Teach Learn Med. 2013; 25(2): 165-170. https://doi.org/10.1080/10401334.2013. 770741

10. Cole D, Rengasamy E, Batchelor S, Pope C, Riley S, Cunningham AM. Using social media to support small group learning. BMC Med Educ. 2017; 17(1): 1-7. https://doi. org/10.1186/s12909-017-1060-7

11. Stawarz K, Preist C, Coyle D. Use of smartphone apps, social media, and web-based resources to support mental health and well-being: Online survey. J Med Internet Res. 2019; 21(7): 1-14. https://doi.org/10.2196/12546

12. Chong JY, Ching AH, Renganathan $Y$, et al. Enhancing mentoring experiences through e-mentoring: a systematic scoping review of e-mentoring programs between 2000 and 2017. Adv Health Sci Educ. 2020; 25(1): 195-226. https://doi.org/10.1007/s10459-019-09883-8

13. Cheston CC, Flickinger TE, Chisolm MS. Social media use in medical education: a systematic review. Acad Med. 2013; 88(6): 893-901. https://doi.org/10.1097/ACM. 0b013e31828ffc23

14. Whyte W, Hennessy C. Social Media use within medical education: a systematic review to develop a pilot questionnaire on how social media can be best used at BSMS. MedEdPublish. 2017; 6(2): 1-36. https://doi. org/10.15694/mep.2017.000083

15. Sutherland S, Jalali A. Social media as an open-learning resource in medical education: current perspectives. Adv Med Educ Pract. 2017; 8: 369-375. https://doi. org/10.2147/amep.s112594

16. Rose S. Medical student education in the time of COVID-19. JAMA. 2020; 323(21): 2131-2132. https://doi. org/10.1001/jama.2020.5227

17. Goh P-S, Sandars J. A vision of the use of technology in medical education after the COVID-19 pandemic. MedEdPublish. 2020; 9(1): 1-8. https://doi.org/10.15694/ mep.2020.000049.1 
18. Merchant RM, Lurie N. Social media and emergency preparedness in response to novel coronavirus. JAMA. 2020; 323(20): 2011-2012. https://doi.org/10.1001/ jama.2020.4469

19. Universidad Continental. 2021. Sílabo de Promoción de la Salud Determinantes [Internet]. Lima: Universidad Continental; 2021 [Consultado 2021 Set 28]. Disponible en: https://repositorio.continental.edu.pe/handle/20.500.12394/9859

20. Asociación Médica Mundial. Declaración de Helsinki de la ANM - Principios éticos para las investigaciones médica en seres humanos [Internet]. Ferney-Voltaire: Asociación Médica Mundial; 2017 [Consultado 2021 Set 28]. Disponible en: https://www.wma.net/es/policies-post/ declaracion-de-helsinki-de-la-amm-principios-eticos-para-las-investigaciones-medicas-en-seres-humanos/

21. Curioso WH, Alvarado-Vásquez E, Calderón-Anyosa R. Usando Twitter para promover la educación continua y la investigación en salud en el Perú. Rev Peru Med Exp Salud Publica. 2011; 28(1): 163-64.

22. Curioso WH, Carnero AM. Promoviendo la investigación en salud con Twitter. Rev Med Hered 2011; 22(3): 121-130.

23. Zhu C, Xu X, Zhang W, Chen J, Evans R. How Health Communication via TikTok Makes a Difference: A Content Analysis of TikTok Accounts Run by Chinese Provincial Health Committees. Int J Environ Res Public Health. 2019; 17(1): 192. https://doi.org/10.3390/ijerph17010192

24. Chen Z, He Q, Mao Z, Chung HM, Maharjan S. A study on the characteristics of douyin short videos and implications for edge caching. ACM DL. 2019 May; 13: 1-6. https://doi.org/10.1145/3321408.3323082

25. Yang S, Zhao Y, Ma Y. Analysis of the Reasons and Development of Short Video Application-Taking TikTok as an Example. En: Proceedings of the 2019 9th International Conference on Information and Social Science (ICISS 2019), Manila, Philippines, 12-14 July 2019.

26. Heldman AB, Schindelar J, Weaver JB. Social Media Engagement and Public Health Communication: Implications for Public Health Organizations Being Truly "Social". Public Health Rev. 2013, 35(1): 1-18.

27. Deng Z, Hong Z, Zhang W, Evans R, Chen Y. The Effect of Online Effort and Reputation of Physicians on Patients' Choice: 3-Wave Data Analysis of China's Good Doctor Website. J. Med. Internet Res. 2019, 21, e10170. doi: 10.2196/10170

28. George DR, Dellasega C. Use of social media in graduate-level medical humanities education: two pilot studies from Penn State College of Medicine. Med Teach. 2011; 33(8): e429-34. doi: 10.3109/0142159X.2011.586749

29. Ranney ML, Genes N. Social media and healthcare quality improvement: A nascent field. BMJ Qual. Saf. 2016; 25: 389-391. doi:10.1136/bmjqs-2015-004827
30. Tengilimoglu D, Sarp N, Yar CE, Bekta s M, Hidir MN, Korkmaz E. The consumers' social media use in choosing physicians and hospitals: The case study of the province of Izmir: Choosing Physicians and Hospitals. Int. J. Health Plan. Manag. 2017; 32: 19-35. doi: 10.1002/hpm.2296

31. Richter JP, Muhlestein DB, Wilks CE. Social media: How hospitals use it, and opportunities for future use. J. Healthc. Manag. 2014, 59, 447-460. PMID: 25647968.

32. Zhang W, Deng Z, Evans R, Xiang F, Ye Q, Zeng R. Social Media Landscape of the Tertiary Referral Hospitals in China: Observational Descriptive Study. J. Med. Internet Res. 2018; 20: e249. doi: 10.2196/jmir.9607

33. Syed-Abdul S, Fernandez-Luque L, Jian W-S, Li Y-C, Crain $\mathrm{S}$, Hsu M-H, Wang Y-C, Khandregzen D, Chuluunbaatar E, Nguyen PA. Misleading Health-Related Information Promoted Through Video-Based Social Media: Anorexia on YouTube. J. Med. Internet Res. 2013; 15: e30. doi: 10.2196/jmir.2237

34. Smailhodzic E, Hooijsma W, Boonstra A, Langley DJ. Social media use in healthcare: $A$ systematic review of effects on patients and on their relationship with healthcare professionals. BMC Health Serv. Res.2016 Ago 26, 16(1):442. doi: 10.1186/s12913-016-1691-0

35. Baron RJ, Berinsky AJ. Mistrust in Science - A Threat to the Patient - Physician Relationship. N. Engl. J. Med. 2019 Jul 11; 381, 182-185. doi: 10.1056/NEJMms1813043

36. Curioso WH. Redes Sociales en Internet: Implicancias para estudiantes y profesionales en salud. Rev Med Hered. 2011; 22(3): 95-7. doi: 10.20453/rmh.v22i3.1081

37. Househ, M.; Borycki, E.; Kushniruk, A. Empowering patients through social media: The benefits and challenges. Health Inform. J. 2014 Mar; 20(1): 50-58. doi: $10.1177 / 1460458213476969$

38. Connelly BL, Certo ST, Ireland RD, Reutzel CR. Signaling Theory: A Review and Assessment. Journal of Management.2011;37(1):39-67.doi:10.1177/0149206310388419

39. MedlinePlus. Biblioteca Nacional de Medicina de los EE.UU. [Internet]. Bethesda: U.S. National Library of Medicine [Consultado 2021 Set 28]. Disponible en: https:// medlineplus.gov/spanish/.

40. Giraldo GA, Gómez MM, Giraldo CF. COVID-19 y uso de redes sociales virtuales en educación médica. Educación Médica. 2021; 22(5): 273-277. https://doi.org/ 10.1016/j. edumed.2021.05.007

41. Curioso WH, Henríquez-Suarez M, Espinoza-Portilla E. Desde Alma-Ata al ciudadano digital: hacia una atención primaria en salud digitalizada en el Perú. Rev Peru Med Exp Salud Publica. 2018; 35(4): 678-683. doi: 10.17843/ rpmesp.2018.354.3710

42. Lupton D. Health promotion in the digital era: a critical commentary. Health Promot Int. 2015 Mar; 30(1): 17483. doi: 10.1093/heapro/dau091 